\title{
Desenvolvimento e avaliação do protótipo da aplicação GISSA ChatBot Mamãe-Bebê para promoção da saúde infantil
}

\author{
Development and evaluation of the GISSA Mother-Baby ChatBot \\ application in promoting child health
}

\author{
Ivana Cristina de Holanda Cunha Barreto (https://orcid.org/0000-0001-8447-3654) ${ }^{1}$ \\ Nardelli Brenda Soares Barros (https://orcid.org/0000-0001-7200-6274) ${ }^{2}$ \\ Rebecca Lucena Theophilo (https://orcid.org/0000-0003-3419-423X) ${ }^{1}$ \\ Vielceketlin Franco Viana (https://orcid.org/0000-0002-3537-1931) ${ }^{1}$ \\ Francisca Raquel de Vasconcelos Silveira (https://orcid.org/0000-0001-7445-605X) ${ }^{3}$ \\ Osvaldo de Souza (https://orcid.org/0000-0003-3577-5538) ${ }^{4}$ \\ Fábio José Gomes de Sousa (https://orcid.org/0000-0003-2168-9647) ${ }^{3}$ \\ Antônio Mauro Barbosa de Oliveira (https://orcid.org/0000-0002-7898-9440) ${ }^{5}$ \\ Luiz Odorico Monteiro de Andrade (https://orcid.org/0000-0002-3335-0619) ${ }^{1}$
}

${ }^{1}$ Fundação Oswaldo Cruz Ceará (Fiocruz Ceará). R. São José s/n, Precabura. 61760-000 Eusébio CE Brasil.

ivana.barreto@fiocruz.br ${ }^{2}$ Programa de Pós-

Graduação em Saúde Pública, Universidade Federal do Ceará (UFC) Fortaleza CE Brasil.

${ }^{3}$ Instituto Federal do Ceará. Campus Maracanau CE Brasil.

${ }^{4}$ Universidade Federal do Ceará. Fortaleza CE Brasil.

${ }^{5}$ Instituto Federal do Ceará. Campus Aracati CE Brasil.
Abstract This study aimed to develop a prototype of the GISSA Mother-Baby ChatBot Application (GCBMB), a conversational agent to promote child health and evaluate the experience of use and satisfaction with this technological solution. This is a two-stage cross-sectional research with a mixed methodology. The first stage develops the settings of dialogue and the GCBMB prototype. The second stage evaluates the experience of using the ChatBot through a structured questionnaire where statements are used to assess the respondent's level of agreement using the Likert Scale and analyzes the application's use path through its database. The sample consisted of 142 puerperae, with a mean age of 25.4 years, where $38.1 \%$ were primiparous. The level of agreement of women with simplicity, good quality of information, clarity of content, usefulness, and satisfaction with the application was above 90\%. Women between 26 and 30 years of age had a higher mean number of hits (5.21), settings accessed (9.26), and usage time (272 seconds) comparing younger and older women. The use of ChatBots on smartphones is encouraging to promote the health of children in Brazil. However, more investments are required to improve technological solutions and research with robust methodologies to evaluate their effectiveness.

Key words Health Strategies, Maternal and Child Health, Digital Health, Public Health, Mobile Applications
Resumo O estudo objetivou desenvolver um protótipo da Aplicação GISSA ChatBot Mamãe-Bebê (GCBMB), um agente conversacional voltado à promoção da saúde infantil, assim como avaliar a experiência de uso e a satisfação com a referida solução tecnológica. Trata-se de uma pesquisa transversal, de metodologia mista, em duas etapas: a $1^{a}$ de desenvolvimento dos cenários de diálogo e do protótipo do GCBMB, e, a 2a de avaliação da experiência do uso do ChatBot por meio de um questionário estruturado, e análise do percurso de uso da Aplicação através de seu banco de dados. A amostra foi de 142 mulheres puérperas, com idade média de 25,4 anos, onde $38,1 \%$ eram primíparas. O nivel de concordância das mulheres com a simplicidade, boa qualidade da informação, clareza do conteúdo, utilidade e satisfação com a aplicação, estiveram acima de $90 \%$. Mulheres entre 26 a 30 anos apresentaram maiores médias de quantidade de acessos (5,21), quantidade de cenários acessados $(9,26)$ e tempo de uso (272 segundos) comparando-se as mais jovens e as mais velhas. O uso de ChatBots em smartphones é animador para promoção da saúde das crianças, porém são necessários mais investimentos para o aperfeiçoamento de soluções tecnológicas e pesquisas com metodologias robustas para avaliar a sua efetividade.

Palavras-chave Estratégias de Saúde, Saúde Materno-Infantil, Saúde Digital, Saúde Pública, Aplicativos Móveis 


\section{Introdução}

Em 1997, a Organização Mundial da Saúde (OMS) reuniu os Estados-Membros para discutir sobre os desafios que a comunidade mundial de saúde enfrentava. Na ocasião, foram considerados os avanços da Tecnologia da Informação e Comunicação (TIC) e definido a Telemática em Saúde como sendo um termo composto para atividades, serviços e sistemas relacionados à saúde, realizados por meios da TIC para fins de promoção global da saúde, controle de doenças e cuidados de saúde, bem como educação, gestão e pesquisa em saúde ${ }^{1}$.

O uso econômico e seguro da TIC em apoio a saúde e as áreas relacionadas à saúde, incluindo serviços, vigilância, literatura, educação, conhecimento e pesquisa foi definido como sendo eSaúde (eHealth) pela Organização Mundial da Saúde em $2005^{2}$.

Eric Topol em 2011 afirmou que as tecnologias digitais, redes sociais, conectividade móvel e a banda larga iriam aumentar o poder da computação e o universo de dados convergiriam com sensores sem fio, genômica, imagem e sistemas de informações de saúde para descontruir com criatividade a medicina até então conhecida. Ele se referia a isso como sendo a saúde digital ${ }^{3}$.

A OMS em 2020 passou a definir Saúde Digital como o campo de conhecimento e prática associado ao desenvolvimento e uso das tecnologias digitais para melhorar a saúde. A saúde digital expande o conceito de eSaúde para incluir os consumidores digitais, com uma maior variedade de dispositivos inteligentes e equipamentos conectados. Também abrange outros usos das tecnologias digitais para a saúde, como a Internet das coisas, inteligência artificial, big data e robótica $^{4}$.

Assim, a mHealth surge como uma subdivisão da saúde digital, a partir da generalização da internet via dispositivos móveis, e, com o avanço da TIC, o surgimento de aplicações móveis para o auxílio e avaliação contínua dos hábitos saudáveis, da autogestão de condições crônicas e outras vertentes, tem aumentado ${ }^{5}$.

O Brasil é signatário da Agenda 2030 da Organização das Nações Unidas $(\mathrm{ONU})^{6}$ na qual existe um consenso entre os estados-membros de que o acesso à internet é um pré-requisito para o desenvolvimento humano na sociedade atual, sendo as TICs uma ferramenta com implicações sociais importantes, no que diz respeito a diminuição das desigualdades e ampliando as possibilidades de diálogo, aprendizado e participação.
Segundo a Pesquisa TIC Domicílios 2019, 99\% dos brasileiros têm o celular como o dispositivo mais usado para acessar a internet, somente um a cada quatro indivíduos não usa a internet, o Nordeste é a região com menor percentual de domicílios com acesso a internet (65\%) e 47\% buscaram informações sobre saúde na internet, percentual que vem crescendo a cada ano da pesquisa ${ }^{7}$.

A internet tem um potencial de democratizar o conhecimento, liberdade pela conexão em rede e liberdade de expressão, dessa forma, os avanços da TIC para promoção da saúde trazem ideias de ações preventivas, a fim de evitar o surgimento de doenças específicas, reduzindo sua incidência e prevalência através do conhecimento epidemiológico, podendo apoiar um processo de educação da população para saúde. Neste sentido, as crianças vêm sendo destaque em muitos estudos e nas políticas públicas, devido sua vulnerabilidade e por considerar que, ao atender suas necessidades de saúde, será mais provável ela atingir a idade adulta com maior qualidade de vida, o que tem repercussões positivas sobre toda a sociedade ${ }^{8}$.

Assim, a implementação de ações voltadas para o bem-estar da criança é um desafio para o sistema de saúde e é um processo em construção, visto que envolve inúmeras ações coletivas e intersetoriais, baseando-se na promoção de saúde com novas práticas e tecnologias, constituindo uma atenção mais integrada e humanizada ${ }^{8}$.

Quão mais específica à informação que se possui, melhor a decisão que poderá ser tomada pelo usuário. Dessa forma, é imprescindível desenvolver ferramentas tecnológicas que tornem mais eficiente o cuidado em saúde de uma forma geral, e no enfoque do presente artigo, na saúde infantil.

A fala é a forma mais natural de comunicação e troca de informações entre seres humanos ${ }^{9}$. As soluções que usam agentes conversacionais (AC) na saúde são tecnologias contemporâneas em ascensão ${ }^{10}$. O aproveitamento de AC para a comunicação em saúde poderia sistematicamente realizar tarefas de diálogo enquanto reduz a carga de comunicação dos serviços de saúde e complementa a entrega de informações de saúde, especificamente relacionadas à promoção de saúde da criança ${ }^{11}$.

O Instituto Atlântico, empresa tecnológica privada sem fins lucrativos, em parceria com o Instituto Federal do Ceará (IFCE), a Universidade Federal do Ceará (UFC), a Fundação Oswaldo Cruz do Ceará (Fiocruz/Ceará), e a startup AVICENA, desenvolveram no contexto da pla- 
taforma tecnológica GISSA ${ }^{12,13}$ o Protótipo da Aplicação GISSA ChatBot Mamãe-bebê, um AC que utiliza técnicas computacionais com regras de decisão para definir o fluxo de interação de diálogos textuais com mães de crianças de 0 a 2 anos de idade sobre cuidados de alimentação, imunização, promoção do crescimento e desenvolvimento.

Dessa forma, o objetivo do estudo foi desenvolver um protótipo da Aplicação GISSA ChatBot Mamãe-Bebê (GCBMB) para promoção da saúde infantil, assim como avaliar a experiência de uso e a satisfação com a referida aplicação por mães de crianças recém-nascidas.

\section{Métodos}

Trata-se de uma pesquisa transversal de metodologia mista em duas etapas ${ }^{14}$ : a $1^{\text {a }}$ de desenvolvimento da aplicação GCBMB, incluindo o desenvolvimento dos cenários de diálogos e o do código computacional, e, a 2a , de avaliação da experiência do uso do protótipo do Agente Conversacional GISSA ChatBot Mamãe-Bebê (Figura 1).

\section{Primeira etapa}

Essa etapa se estruturou de janeiro a setembro de 2019, sendo executada por uma equipe interdisciplinar envolvendo profissionais da saúde (médicos, enfermeiros, psicólogos, assistente sociais) e da tecnologia (engenharia da computação e especialistas em Programação de Linguagem Natural - PLN).

\section{Desenvolvimento dos cenários}

Uma equipe interdisciplinar de profissionais da área da saúde e profissionais da tecnologia da informação construíram cenários de possíveis diálogos sobre cuidados de alimentação, imunização, promoção do crescimento e desenvolvimento do bebê de 0 a 2 anos de idade, totalizando 15 cenários. As informações para criação dos cenários foram extraídas dos Cadernos de Atenção Básica do Ministério da Saúde referentes à Atenção à Saúde do Recém-nascido ${ }^{15,16}$, Saúde da Criança: aleitamento materno e alimentação complementar ${ }^{17}$, Pré-natal de Baixo Risco ${ }^{18}$, do Manual de Normas e Procedimentos para Imunização, incluindo o Calendário Vacinal Atualizado $^{19}$.

A Figura 2, presente na seção dos Resultados, apresenta o Cenário 1, que dispõe de orientações sobre amamentação para bebês de até seis meses, que posteriormente foi adicionado a uma base de dados e utilizado pelo GISSA ChatBot Mamãe -Bebê para conversação com as usuárias.

\section{Desenvolvimento da aplicação GISSA ChatBot Mamãe-Bebê}

É importante registrar que o GISSA Cha$t B o t$, além do módulo direcionado para mães ou cuidadores de crianças menores de dois anos, avaliado no presente estudo, possui outros dois módulos: um direcionado para gestantes com orientações sobre intercorrências na gravidez, e um terceiro para avaliação da satisfação de usuários de serviços de saúde.

O frontend do Gissa Chatbot foi desenvolvimento usando o framework React Native, uma ferramenta javascript que desenvolve aplicativos mobile nativos para iOS e Android ${ }^{20}$. A interface do bot é constituída de algumas funcionalidades como: login de acesso ao sistema por meio do perfil do facebook, facilitando o acesso aos usuários, para isto foi usado a ferramenta firebase; interação entre os perfis dos usuários com o sistema, por meio de telas e botões personalizados com interface amigável, e a personagem virtual "Helena da Saúde", conforme os padrões de usabilidade; implementação do consumo de serviços de uma API (Application Programming Interface) disponibilizada pelo backend para comunicação com o servidor.

O backend foi desenvolvido usando a linguagem de programação Python com a tecnologia Flask $^{21}$. Foi implementado um modelo de esquemas de fluxos que possibilitou a inserção dos cenários desenvolvidos pela equipe de saúde. Este esquema gerou uma tabela com perguntas e respostas. Cada resposta pode levar a um novo fluxo de conversa e assim, sucessivamente, até encerrar o diálogo. Foi usada a biblioteca NLTK, com processamento básico de linguagem natural, que permite interpretar textos básicos digitados pelo usuário, como "s" para "sim" e "n" para "não". Foi implementado ainda uma API http que permite a comunicação com o frontend, possibilitando o consumo destes serviços pelo usuário do Chatbot $t^{22}$.

\section{Segunda etapa}

Avaliação da experiência de uso da Aplicação GISSA ChatBot Mamãe-Bebê: a população constituiu-se por mães de recém-nascidos com pelo menos 24 horas de vida alocadas no Alojamento Conjunto $^{15}$ do Hospital Nossa Senhora da Conceição (HNSC) em Fortaleza-CE. Foram excluí- 


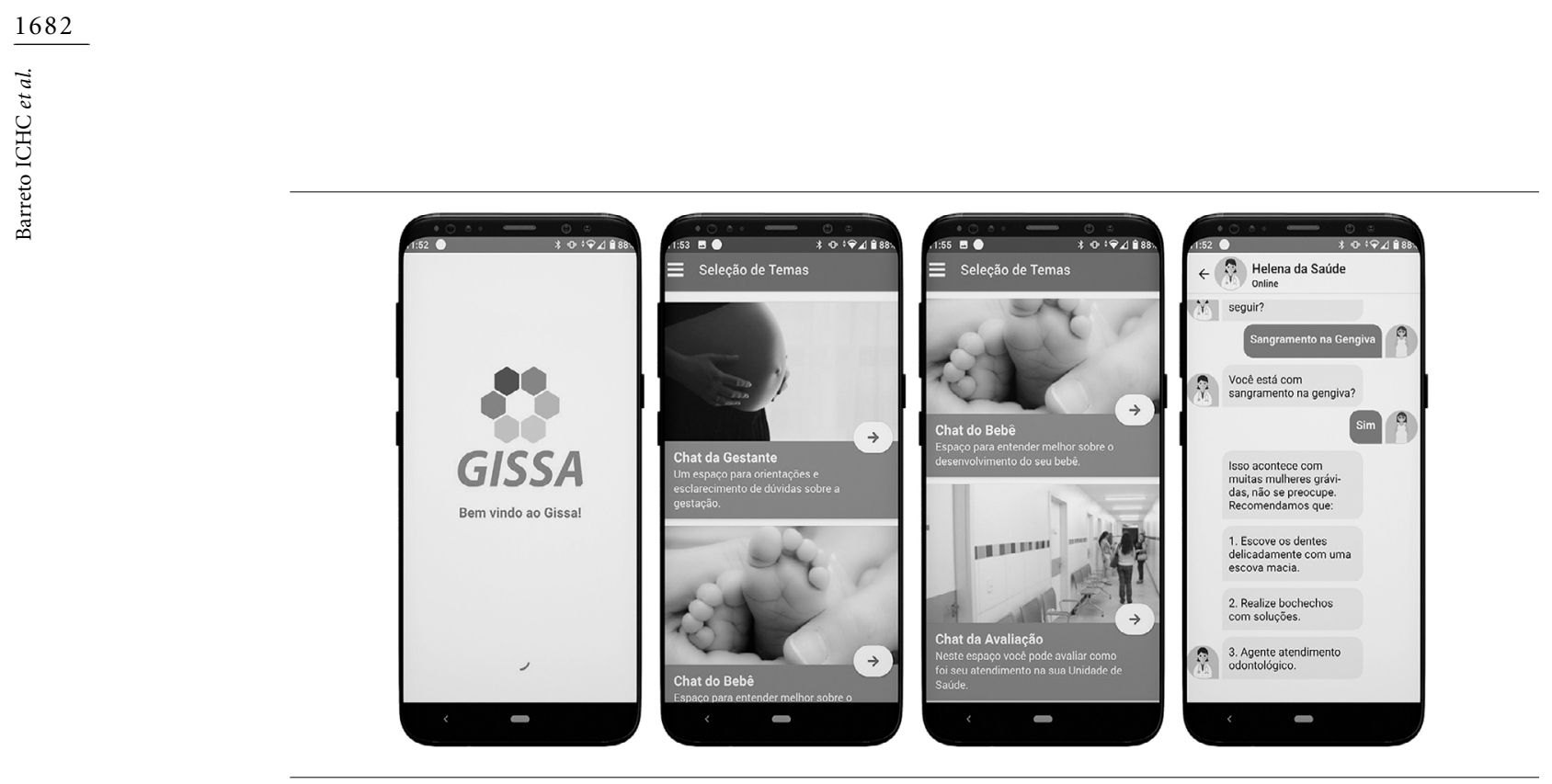

Figura 1. Demonstração do GISSA ChatBot Mamãe-Bebê (telas iniciais).

Fonte: Elaborada pelos autores.

das as mães de recém-nascidos que não houvessem completado 24 horas de vida e as menores de 18 anos. A coleta aconteceu em dias alternados, de 22 outubro a 29 novembro de 2019.

No total, foram abordadas 210 mulheres, sendo 7,6\% ( $\mathrm{n}=16)$ excluídas por terem menos de 18 anos, e $24,7 \%(n=52)$ se recusado a participar da pesquisa. A amostra final foi de 142 mães de recém-nascidos, sendo a mesma representativa da população de 240 que deram à luz no período do estudo no Hospital Nossa Senhora da Conceição, com grau de confiança de $90 \%$ e margem de erro de $5,3 \%$.

\section{Estudo avaliativo sobre experiência de uso e satisfação com o protótipo}

O protótipo da aplicação GISSA ChatBot Mamãe-Bebê, instalado em um celular de uso da pesquisa, foi apresentado às mães, que manusearam livremente o protótipo conforme seu interesse nos assuntos disponibilizados por idade do bebê, tais como: amamentação, introdução alimentar, imunização e marcos do crescimento e desenvolvimento.

Após o manuseio do GISSA ChatBot Mamãe -Bebê, o questionário estruturado de avaliação foi preenchido pela pesquisadora, com questões sobre a identificação da mãe, escolaridade, histórico gestacional, informações sobre o nascimento do bebê. Para avaliação do protótipo foi feita a pergunta "Em relação a sua experiência com a
Aplicação Mamãe-Bebê, o quanto você concorda com as afirmativas abaixo?", seguidas das seguintes afirmativas: O GCBMB é simples de usar; O GCBMB possui as informações que preciso; Entendi bem os comandos do GCBMB; O GCBMB tem palavras simples; O GCBMB me ajudou a cuidar melhor do meu bebê; Eu gostei de usar o GCBMB; Eu gostei da aparência do GCBMB; Eu pretendo usar o GCBMB no meu dia-a-dia.

As respostas eram psicométricas, baseadas na Escala de Likert compreendendo as seguintes alternativas de resposta: concordo totalmente, concordo parcialmente, nem concordo e nem discordo, discordo parcialmente, discordo totalmente.

\section{Análise do percurso de manuseio do protótipo}

Através da mineração dos dados oriundos da base de dados do GISSA ChatBot Mamãe-Bebê, essa sub etapa analisou o percurso de manuseio das participantes no uso da aplicação, como por exemplo: quantidade de acessos, quais cenários e assuntos foram mais acessados, tempo de uso médio em cada cenário.

Após concluir a análise estatística dos dados coletados no questionário quantitativo, em que foi observado um grau de concordância das participantes com as questões de avaliação do ChatBot acima de 95\%, os autores decidiram realizar algumas entrevistas abertas, para compreender melhor o porquê de tão elevada concordância, e 


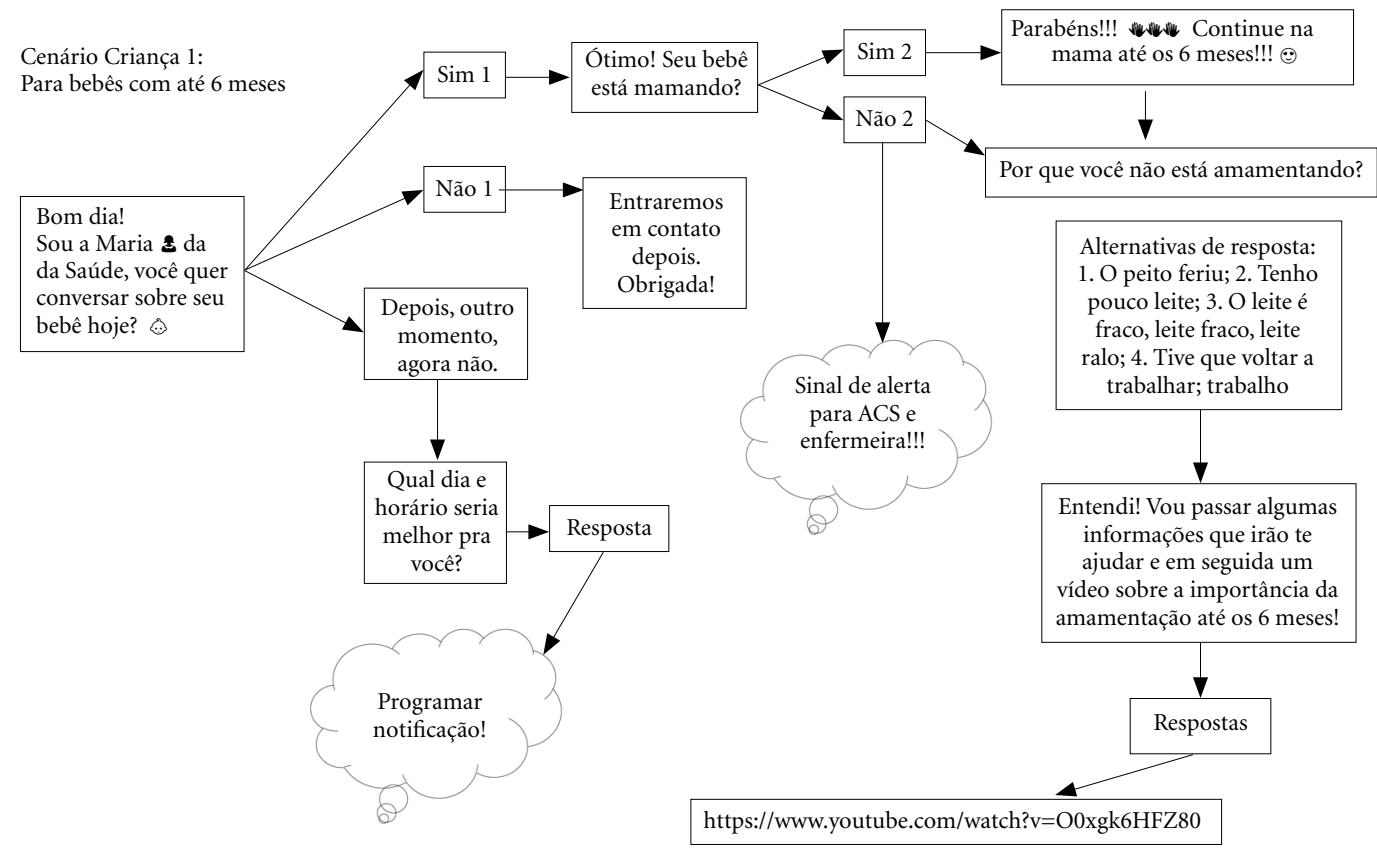

Figura 2. Demonstração de um Cenário construído para orientações sobre amamentação de crianças de até seis meses.

Fonte: Elaborada pelos autores.

RESPOSTA 1: Se o bico do peito feriu, pode ser um desafio muito grande na amamentação, a dor pode causar desânimo pra você. Mas se seu desejo é voltar a amamentar devido a grande importância que tem para seu bebê, não desista. Peça ajuda ao seu agente de saúde e a(o) enfermeira(o) da sua área. Obrigada pela atenção!

RESPOSTA 2: Vou passar algumas dicas para aumentar a produção do seu leite: Tenha a certeza que seu bebê está fazendo a pega corretamente (se necessário peça ajuda a um profissional); Deixe o bebê mamar sempre que ele quiser; Tenha sempre o contato pele a pele com o bebê por mais tempo; Espere o bebê soltar o peito naturalmente; Beba bastante água, pois a hidratação é importante para produção de leite! Espero ter contribuído! Obrigada pela atenção!

RESPOSTA 3: Não existe leite materno fraco! A qualidade do seu leite é ideal para o bebê, sua produção é suficiente e adequada para seu filho até os 6 meses, ok? Seu leite tem digestão mais rápida, por isso o bebê quer mais vezes seu peito! Além disso seu leite contém substâncias de proteção que são essenciais para seu bebê!! Espero ter ajudado! Obrigada pela atenção!

RESPOSTA 4: Você sabia que seu leite pode ser armazenado tanto na geladeira como no congelador de sua casa em um recipiente de vidro? O prazo de validade do leite cru é de 12 horas se guardado na geladeira e de 15 dias se estocado no freezer ou congelador. Qualquer dúvida entre em contato com agente de saúde da sua área ou a(o) enfermeira (o)! Espero que tenha ajudado! Obrigada pela atenção!

Possibilidades de sinônimos das palavras Sim e Não:

\begin{tabular}{|l|l|}
\hline SIM 1 & $\begin{array}{l}\text { Pode ser; Tudo bem; Posso; Com certeza; Claro; } \\
\text { Quero; Humrum. }\end{array}$ \\
\hline NÃO 1 & Quero não; Não posso; quero nao; nao; poso nao \\
\hline SIM 2 & $\begin{array}{l}\text { Desde que nasceu; Nunca parou; Claro; Com } \\
\text { certeza; Humrum. }\end{array}$ \\
\hline NÃO 2 & Nunca mamou; nao \\
\hline
\end{tabular}

OBS DO VÍDEO:

\begin{tabular}{|l|c|c|}
\hline \multicolumn{1}{|c|}{ Link do vídeo } & $\begin{array}{c}\text { Início } \\
\text { do vídeo } \\
\text { (min) }\end{array}$ & $\begin{array}{c}\text { Fim do } \\
\text { vídeo } \\
\text { (min) }\end{array}$ \\
\hline $\begin{array}{l}\text { https://www.youtube.com/ } \\
\text { watch?v=O0xgk6HFZ80 }\end{array}$ & $0: 09 \mathrm{~s}$ & $02: 45 \mathrm{~s}$ \\
\hline
\end{tabular}

afastar possíveis vieses, como por exemplo: o desejo da participante de agradar a entrevistadora ou de finalizar logo o momento de entrevista.

As entrevistas abertas aconteceram no AC do HNSC tendo fluxo metodológico inicial com aplicação do questionário estruturado de avaliação do uso. Após este momento a participante era informada da necessidade da gravação de áudio para continuidade da coleta, reforçando nesse ponto se ela possuía desejo de continuar com sua 
participação, a seguir sendo aplicadas perguntas abertas sobre a utilização do ChatBot.

\section{Observação direta do uso do aplicativo pelos pesquisadores}

Essa sub etapa foi criada para ser realizada um momento de avaliação da percepção dos pesquisadores, como também, uma reflexão crítica sobre a aplicação e seu uso, se foi percebida alguma dificuldade não reportada pela participante, como também a avaliação do manuseio dos smartphones, em geral, levando em conta a diversidade social e educacional da população assistida no HNSC.

\section{Análise dos dados}

Os dados do estudo quantitativo foram organizados e analisados no Stata Package Statistics versão 13 para desktop. Foram aplicadas análises descritivas, e análises bivariadas utilizando o teste estatístico T test de Student.

As entrevistas abertas foram transcritas e analisadas pelas pesquisadoras, buscando compreender os motivos da boa avaliação do GISSA Chatbot Mamãe-Bebê pelas mulheres participantes do estudo.

\section{Aspectos éticos}

A pesquisa foi submetida, através da Plataforma Brasil, ao Comitê de Ética em Pesquisa da Escola de Saúde Pública do Ceará (ESP/CE) e foi aprovada. Todas as entrevistadas foram esclarecidas sobre a finalidade e a importância da pesquisa e assinaram o Termo de Consentimento Livre e Esclarecido (TCLE).

\section{Resultados}

\section{Caracterização do GCBMB}

O GCBMB é um protótipo de aplicação mobile que utiliza as linguagens de programação Python e Javascript e as tecnologias react native, flesk e firebase. Ele é composto por três módulos, presentes na tela inicial da aplicação, a saber Chat da Gestante, Chat do Bebê e Chat da Avaliação do serviço de saúde, sendo o Chat do Bebê o alvo do presente estudo.

Após a escolha pelo Chat do Bebê, o usuário tem acesso a uma tela onde a personagem virtual "Helena da Saúde" cumprimenta-o e pergunta se quer conversar sobre a saúde do seu Bebê. Caso a resposta do usuário seja "Sim", é ofertada uma relação de idades em meses, num rol de 1 a 24 meses de vida. Ao selecionar a idade desejada, o usuário tem acesso a uma sequência de perguntas e, conforme suas respostas, orientações adequadas para a situação específica abordada, como por exemplo: cuidados e orientações sobre problemas na amamentação, introdução a alimentação complementar, checagem do calendário vacinal, marcas do desenvolvimento para a idade selecionada e sinais de risco. As telas iniciais da aplicação são apresentadas na Figura 1, já na Figura 2, é apresentado o desenho de um dos cenários de diálogos textuais do GCBMB, no caso, para orientações sobre amamentação de crianças até 6 meses.

\section{Estudo avaliativo sobre experiência de uso e satisfação com o protótipo}

A média de idade das participantes foi de 25,4 anos, com valor mínimo de 18 e máximo de 40 anos. Das 142 participantes, 68,3\% $(n=97)$ possuíam o ensino médio incompleto ou algum nível de escolaridade superior. Apenas 4,9\% $(n=7)$ não possuíam celular próprio e $8,5 \%(n=12)$ não possuíam acesso à internet pelo celular. Na Tabela 1 são apresentados mais dados sobre o perfil sociodemográfico das puérperas.

Quanto aos antecedentes obstétricos, 33,8\% $(\mathrm{n}=48)$ das mulheres eram primigestas (primeira gestação) e $38,1 \%(n=54)$ primíparas (primeiro parto). $26,1 \%(n=37)$ das participantes possuíam histórico de aborto, sendo que 4,2\% (n=6) delas possuíram 2 ou mais. Com relação a gestação atual, 64,8\% $(n=81)$ das mulheres tiveram sete ou mais consultas de pré-natal, e $57,9 \%(n=81)$ parto cesáreo.

Dentre as intercorrências durante o período gravídico, a de maior prevalência foi a ocorrência de infecção urinária com 46,5\% $(n=66)$, seguida de anemia com 13,4\% $(n=19)$. Apenas $15,5 \%$ $(\mathrm{n}=22)$ das intercorrências referidas pelas puérperas eram preexistentes à gravidez. Outros agravos citados foram hipertensão $(7,7 \% / \mathrm{n}=11)$, diabetes mellitus $(6,3 \% / \mathrm{n}=9)$, sangramento vaginal $(6,5 \% /$ $\mathrm{n}=9)$ e ameaça de parto prematuro $(3,5 \% / n=5)$.

Os recém-nascidos, em sua maioria, nasceram com peso adequado $(83,9 \% / \mathrm{n}=109)$ e foram estimulados à amamentação $(75,4 \% / \mathrm{n}=107)$. $36,6 \%(n=52)$ deles tiveram alguma complicação após o nascimento.

Na Tabela 2 apresentam-se os resultados das questões de avaliação da experiência de uso e satisfação com o GCBMB. O nível de concordância 
Tabela 1. Perfil sociodemográfico das puérperas. Fortaleza, 2019 ( $\mathrm{n}=142)$.

\begin{tabular}{lrr}
\hline \multicolumn{1}{c}{ Característica } & N & $\%$ \\
\hline Idade (faixa etária) & & \\
18 a 29 & 101 & 71,1 \\
30 a 40 & 41 & 28,9 \\
Escolaridade & & \\
$\quad$ Até ensino fundamental completo & 45 & 31,7 \\
$\quad$ Ensino médio incompleto ou + & 97 & 68,3 \\
Possui celular próprio & & \\
$\quad$ Não & 7 & 4,9 \\
$\quad$ Sim & 135 & 95,1 \\
Possui internet no celular & & \\
$\quad$ Não & 12 & 8,5 \\
Sim & 130 & 91,5 \\
\hline
\end{tabular}

Fonte: Elaborada pelos autores.

das mulheres com a simplicidade, boa qualidade da informação, clareza do conteúdo, utilidade e satisfação com a aplicação de uma forma geral, estive acima de $90 \%$.

Cruzou-se as respostas de avaliação do aplicativo com a faixa etária, a escolaridade e a paridade das participantes, não havendo diferenças estatisticamente significativas quanto a satisfação e facilidade de uso (Tabela 3 ).

Algumas sugestões das puérperas foram registradas no estudo avaliativo: incluir mais orientações sobre cuidados com recém-nascidos, como limpeza do coto umbilical e banho; detalhes sobre a amamentação, como ordenha e explicações sobre os tipos de mamilo. Outra sugestão foi de que o aplicativo deveria permitir ao usuário "rolar" a tela sozinho ou diminuir o tamanho dos textos, sendo esta funcionalidade importante em cenários em que o ChatBot apresenta muitas respostas, como dicas sobre a alimentação complementar.

\section{Análise do manuseio do GISSA \\ ChatBot Mamãe-Bebê}

Ao iniciar a conversação com o ChatBot, o usuário é direcionado, primeiramente, a um cenário introdutório. A cada acesso, o usuário pode iniciar e finalizar no cenário introdutório, assim como, pode ser direcionado a outros cenários, conforme as respostas aos diálogos. O usuário também pode interromper a conversação de um cenário com o ChatBot a qualquer momento. Dos 466 acessos analisados, em 129 o usuário in-
Tabela 2. Avaliação de uso do GISSA ChatBot MamãeBebê. Fortaleza, 2019 ( $\mathrm{n}=142)$.

\begin{tabular}{|c|c|c|}
\hline Característica & $\mathbf{N}$ & $\%$ \\
\hline O GCBMB é simples de usar & & \\
\hline Concordo Totalmente & 134 & 94,4 \\
\hline Outras respostas & 8 & 5,6 \\
\hline $\begin{array}{l}\text { O GCBMB possui as informações } \\
\text { que preciso }\end{array}$ & & \\
\hline Concordo Totalmente & 129 & 90,8 \\
\hline
\end{tabular}

139,2

Os comandos do GCBMB são

rápidos

Concordo Totalmente

Outras respostas

13998,6

21,4

Entendi bem os comandos do GCBMB

$$
\text { Concordo Totalmente }
$$

Outras respostas

128

13

90,8

O GCBMB tem palavras simples

Concordo Totalmente

Outras respostas

$134 \quad 95,0$

$7 \quad 5,0$

O GCBMB me ajudou a cuidar

melhor do meu bebê

Concordo Totalmente

Outras respostas

13293,6

96,4

Eu gostei de usar o GCBMB

Concordo Totalmente

Outras respostas

13796,4

$5 \quad 3,6$

Eu gostei da aparência do GCBMB

Concordo Totalmente

Outras respostas

13697,1

$4 \quad 2,9$

Eu pretendo usar o GCBMB no

meu dia a dia

Concordo Totalmente

Outras respostas

13192,9

$10 \quad 7,1$

Ocorreu algum erro ao usar o

GCBMB

\begin{tabular}{lrr} 
Não & 125 & 91,9 \\
Sim & 11 & 8,1 \\
\hline
\end{tabular}

Fonte: Elaborada pelos autores.

terrompeu a conversação de forma abrupta logo no cenário introdutório; em 76 acessos, o usuário interrompeu a conversação depois de ter finalizado pelo menos um cenário; e em 261 acessos, o usuário encerrou o ChatBot após a finalização da conversação de todos os cenários dialogados. Portanto, em 337 acessos, o ChatBot foi encerrado pelo usuário após a conclusão da conversação de, pelo menos, um cenário de diálogo. Desses 337 acessos, 312 trataram a conversação sobre 


\begin{tabular}{|c|c|c|c|c|}
\hline \\
\hline 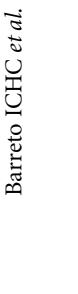 & \multicolumn{4}{|c|}{$\begin{array}{l}\text { Tabela 3. Relação entre pretensão de uso do ChatBot } \\
\text { GISSA Mamãe-bebê por puérperas versus idade, } \\
\text { escolaridade e paridade. Hospital Nossa Sra. da } \\
\text { Conceição, Fortaleza, } 2019 .\end{array}$} \\
\hline & \multirow[t]{2}{*}{ Pretendo usar o app } & \multicolumn{2}{|c|}{$\begin{array}{l}\text { Concordou } \\
\text { totalmente }\end{array}$} & \multirow[t]{2}{*}{ p-valor } \\
\hline & & $\mathrm{N}=131$ & $\%$ & \\
\hline & \multicolumn{4}{|l|}{ Idade } \\
\hline & Até 29 anos & 90 & 68,7 & 0,7009 \\
\hline & 30 anos ou mais & 41 & 31,3 & \\
\hline & \multicolumn{4}{|l|}{ Escolaridade } \\
\hline & $\begin{array}{l}\text { Até ensino } \\
\text { fundamental completo }\end{array}$ & 42 & 32,1 & 0,7275 \\
\hline & $\begin{array}{l}\text { Ensino médio } \\
\text { incompleto ou }+ \\
\text { Quantidade de partos }\end{array}$ & 89 & 67,9 & \\
\hline & Primípara & 52 & 39,7 & 0,9391 \\
\hline & Multípara & 79 & 60,3 & \\
\hline
\end{tabular}

Fonte: Elaborada pelos autores.

criança, permitindo a visualização de cenários de promoção da saúde da criança por 682 vezes.

Considerando que cada usuário pode acessar mais de um cenário a cada acesso, dos 337 acessos realizados ao ChatBot, as conversações abordaram ao todo 796 cenários incluindo os relativos as crianças, gestantes e a avaliação dos serviços, tendo uma média de conversação sobre, aproximadamente, 2 cenários a cada acesso. Os três cenários de maior acesso foram: Cenário 1 (bebês com até 6 meses) com 115 acessos; Cenário 2 (bebê de 2 meses) com 74 acessos; Cenário 7 (bebês com 6 meses enfatizando alimentação complementar) com 21 acessos.

Em relação ao tempo de uso, considerando os 312 acessos que trataram de conversação sobre crianças e os cenários cuja conversação foi concluída sem interrupção, o tempo médio da conversação foi de, aproximadamente, 27 segundos em cada cenário e de, aproximadamente, 48 segundos em cada acesso (incluindo a conversação de todos os cenários dialogados pelo usuário durante o acesso). No que diz respeito a um acesso, o maior tempo de conversação foi de 20 minutos e 52 segundos e o menor tempo de uso foi de 1 segundo.

A seguir são apresentadas informações sobre os cenários de crianças cujas conversações foram mais frequentes: Cenário Criança 1 e Cenário Criança 2.

A conversação sobre a promoção da saúde para bebês com até 6 meses (Cenário Criança 1) inicia perguntando ao usuário se o bebê está mamando. Das 115 conversações nesse cenário, 91 usuários responderam que o bebê está mamando ("Sim"), 15 usuários responderam que o bebê não está mamando ("Não") e 9 usuários interromperam a conversação nessa pergunta. Dos 15 usuários que responderam que o bebê não está mamando, 4 responderam que isso está acontecendo porque o peito feriu, 4 porque tem pouco leite, 1 porque o leite é fraco/ralo e 6 interromperam a conversação sem mencionar o motivo da ausência da amamentação.

A conversação sobre criança de 2 meses (Cenário Criança 2) inicia tratando da vacinação, perguntando se o usuário pode olhar o cartão de vacina, das 74 conversações sobre esse cenário, 11 interromperam a conversação nessa pergunta; para 3 usuários não foi possível olhar o cartão de vacina, finalizando a conversação desse cenário com usuário; e 60 usuários informaram que é possível olhar o cartão de vacina. Para esses, a conversação continua perguntando se o bebê já tomou as 4 vacinas agendadas para os dois meses: 5 interromperam a conversação nessa pergunta, 14 informaram que "Não" e 41 informaram que "Sim". Para quem respondeu "Sim", o ChatBot pergunta se o bebê já olha para a pessoa e fica observando e acompanha objetos: 11 interromperam a conversação nessa pergunta, 13 informam que "Não" e 17 informam que "Sim". Para quem respondeu que o bebê não tomou as vacinas, o ChatBot pergunta se deseja ver um vídeo sobre a importância da vacinação: 2 usuários interromperam a conversação nessa pergunta, 9 responderam que "Sim" e 3 responderam que "Não".

Ressalta-se, ainda, que a conversação sobre um cenário passa por um fluxo de perguntas e respostas entre o usuário e o ChatBot. Como mencionado anteriormente, dos 682 cenários sobre criança abordados nas conversações, em 67 vezes, o usuário interrompeu a conversação logo no cenário introdutório e em 120 vezes, o usuário interrompeu a conversação depois de ter finalizado pelo menos um cenário, ou seja, a interrupção não se deu no cenário introdutório.

Das análises dos dados do manuseio do ChatBot a partir do banco do protótipo agregados aos do estudo avaliativo sobre usabilidade e satisfação dos usuários, não foram observadas diferenças consistentes na média de quantidade de acessos, de cenários acessados e de tempo de uso por nível escolaridade.

Por outro lado, observou-se que as mulheres entre 26 e 30 anos apresentaram maiores médias de quantidade de acessos $(5,21)$, quantidade de 
cenários acessados $(9,26)$ e tempo de uso (272 segundos) comparando-se as mais jovens e as mais velhas.

Em média os acessos tiveram uma duração de 48 segundos, o que pode ser considerada pequena. Como possíveis causas para esta curta duração podem ser consideradas, além de limitações na qualidade do ChatBot, o fato da pesquisa ter sido realizada com puérperas no contexto do alojamento conjunto, sob o estresse puerperal, o que pode ter desestimulado a exploração da aplicação.

Foram realizadas entrevistas abertas com 6 mulheres após o manuseio do ChatBot e a aplicação do questionário sobre a experiência de uso e satisfação. De uma forma geral as respostas das mulheres nesta etapa evidenciaram que elas realmente tiveram facilidade para utilizar e compreender o ChatBot.

Duas participantes relataram já ter utilizado aplicativos para ajudar no cuidado infantil. Uma delas quando pedida para comparar os aplicativos, comentou que o GISSA ChatBot Mamãe-Bebê era mais prático porque "o outro não dava exatamente o que a gente queria: a gente só via né? e aqui ele explica, tem os vídeos, tem as coisas para a gente ver direitinho. $\mathrm{O}$ outro a gente tinha que procurar e ler tudo". Outra participante também relatou que gostou do aplicativo porque ele utilizava fotos e vídeos para complementar a parte escrita.

Contrariamente, durante uma das entrevistas em que a puérpera atribuiu nota máxima a todos os quesitos de usabilidade do ChatBot, foi percebido certa urgência da participante em responder rápido o questionário e quando perguntada sobre o motivo para isso, foi relatado que a mesma havia recebido a alta hospitalar e estava ansiosa para ir para casa. Ela havia dado nota máxima em todos os quesitos de avaliação do aplicativo e, portanto, pode-se notar o peso do fator "pressa" para a participante $\mathrm{N}^{\circ} 1$, "fui só respondendo mesmo" foram as palavras usadas por ela.

As sugestões apresentadas por elas nesta etapa foram: o desenho de um novo ícone, algo que lembrasse mais o cuidado com a criança; que o aplicativo abordasse o desenvolvimento das crianças em idade superior a dois anos; que o aplicativo funcionasse de modo que, periodicamente, ele trouxesse algo novo sobre o cuidado com o bebê ou um alerta.

\section{Discussão}

Este estudo demonstrou a viabilidade de desenvolvimento de soluções tecnológicas aplicadas à saúde no contexto de um ecossistema de inovação composto por equipes interdisciplinares e interinstitucionais.

Os resultados do estudo apontaram que a maioria das mulheres participantes apresentaram facilidade de manuseio, boa compreensão e satisfação no uso do GCBMB. Considerando que metade dessas tinham apenas o ensino fundamental, esses resultados contrariam uma expectativa inicial da equipe de que haveria dificuldades no uso da aplicação, demonstrando uma familiaridade significativa no manuseio de aplicativos em smartphones pela população estudada.

Frente às transformações tecnológicas, a grande prevalência do uso de smartphones já é conhecida e, mesmo nas áreas em que a população tem menor nível socioeconômico, como o da população atendida no Hospital onde foi realizado o presente estudo, a presença dos aparelhos móveis é massiva.

No contexto brasileiro, Spizzirri et al. ${ }^{23}$ pesquisou sobre o uso da tecnologia na adolescência, na chamado "Geração Digital” e, quanto à intensidade da disseminação das tecnologias nessa faixa etária, afirmam que o Brasil é um dos países recordistas em número de usuários residenciais como também no número médio de horas mensais de utilização da internet ${ }^{23}$.

Silva et al. ${ }^{24}$ realizaram um estudo sobre as diferenças de gênero na utilização das TICs e sugerem que, apesar das pesquisas mostrarem que existe certa equidade de gênero no acesso à internet, as mulheres se relacionam diferente dos homens com as TICs e que, portanto, à médio -longo prazo isso deve impactar as perspectivas da mulher na sociedade da informação e do conhecimento ${ }^{24}$.

Essa perspectiva agregada com os papéis de gênero ainda enraizados na sociedade, sugerem que a mulher use as TICs relacionadas à saúde não apenas para seu autocuidado, mas também o de sua família ${ }^{25}$.

A adequação do Chatbot às realidades de seu público-alvo também é uma grande vantagem. A possibilidade de ter uma inovação tecnológica resolutiva e de baixo custo que trabalha com problemas enfrentados na atenção primária à saúde 
é animadora. Isso, principalmente, quando se trata da disseminação de informações de cuidado em saúde através de um dispositivo que a grande maioria da população possui: o smartphone.

A adequabilidade de linguagem e a utilização de vídeos explicativos como uma ferramenta visual são exemplos que essa inovação traz para facilitar a transmissão dos conhecimentos, sendo uma forma inovadora da prática de promoção da saúde, que pode e deve atuar aliada a atenção básica, por meio da Estratégia Saúde da Família (ESF) no Brasil.

A partir desse enfoque, a aplicabilidade da ferramenta ChatBot na atenção à saúde é reafirmada e atualmente essa ferramenta já existe para os mais diversos temas. Alguns exemplos: Kumar e Keerthana ${ }^{26}$ trazem a proposta de um agente de saúde virtual com quem os usuários possam sanar suas dúvidas de saúde; Ilić e Markovićc ${ }^{27}$ fizeram uma revisão sobre o uso da Inteligência Artificial (IA) na saúde, apontando diminuição nos custos dos serviços; Cameron et al. ${ }^{28}$ trazem a proposta da criação de um ChatBot para aconselhamento virtual em saúde mental.

Apesar desse tipo de programa permitir sua adoção em diversos temas na área da saúde, é importante ressaltar que, atualmente, o uso do ChatBot ainda é limitado quando utilizado dentro da saúde pública, sendo necessários mais investimentos em melhorias a nível de design e segurança, por exemplo ${ }^{11}$.

O uso da ferramenta na atenção materno-infantil é mais frequente durante o período gravídico. Yadav et al. ${ }^{29}$ trouxeram um estudo sobre a oportunidade do uso de ChatBots na educação sobre aleitamento materno na Índia, trazendo o exemplo de algumas das aplicações que abordam o pós-gravidez nesse assunto.

Nesse panorama, segundo os resultados de boa usabilidade e satisfação com o GISSA ChatBot Mamãe-Bebê, pode-se concluir de que o aplicativo quando utilizado, tem potencialidade para causar impacto positivo no cuidado com crianças na primeira infância, despertando a atenção das mães aos marcos do crescimento e desenvolvimento, alimentação adequada e as imunizações necessárias.
Dentre as limitações percebidas durante o estudo, pode-se citar que seria interessante ter abordado mães de crianças entre um e vinte e quatro meses, o que deverá ser realizado em futuros estudos. Outro aspecto a ser comentado, é que a avaliação do uso da aplicação por meio de um estudo longitudinal poderá trazer mais conhecimentos sobre sua usabilidade e a adesão dos usuários. É preciso justificar que a aplicação ainda está na fase de prototipação e deverá ser melhor desenvolvida e avaliada para incremento de sua efetividade.

A partir da experiência das pesquisadoras na observação do uso da aplicação pelas puérperas faz-se as seguintes sugestões de melhoria: mostrar na parte inicial do que se trata a aplicação, e para isso sugere-se escolher uma logo com algo que faça referência à gravidez e ao recém-nascido; retornar a usuária ao menu de cenários disponíveis quando ela acionar a opção "voltar"; redirecionar a usuária para reiniciar o uso quando os vídeos sugeridos encerrarem; agregar tecnologia de inteligência artificial para possibilitar novas respostas as perguntas feitas pelas mulheres por meio da aplicação.

É importante ressaltar a originalidade do presente estudo no Brasil, sendo que não foram identificadas na literatura nacional, e da América Latina, publicações que relatem avaliações de experiências de uso de agentes conversacionais nos serviços de saúde ${ }^{11}$.

Estes resultados demonstram que a utilização da ferramenta de ChatBot na plataforma de smartphones é animadora para promoção da saúde das crianças. Entretanto, são necessários investimentos significativos para desenvolver ferramentas de qualidade, avaliar a efetividade e monitorar os resultados de sua utilização.

O campo da tecnologia, especificamente da Saúde Digital, está em constante e rápida evolução e acredita-se que a tecnologia do Chatbot, como também o seu uso dentro das entidades de saúde, avançará em conjunto. Para isso, são necessários mais estudos, principalmente longitudinais, que possam analisar e reportar com mais acurácia a utilização do ChatBot dentro da Saúde Pública. 


\section{Colaboradores}

ICHC Barreto trabalhou na concepção, na pesquisa e na metodologia, na redação do texto e na revisão final. NBS Barros trabalhou na pesquisa $\mathrm{e}$ na redação do texto. RL Theophilo trabalhou na pesquisa e na redação do texto. VF Viana trabalhou na pesquisa e na redação do texto. FRV Silveira trabalhou na pesquisa, na redação do texto e na revisão final. O Souza trabalhou na pesquisa. FJG Sousa trabalhou na pesquisa e na redação do texto. AMB Oliveira trabalhou na pesquisa. LOM Andrade trabalhou na pesquisa, na redação do texto e na revisão final do artigo.

\section{Referências}

1. World Health Organization (WHO). Health-for-all policy for the twety-first century: "health telematics". Executive Board. Geneva: WHO; 1998.

2. World Health Organization (WHO). WHO eHealth Resolution [Internet]. 2005 [acessado 2020 mar 24]. Disponível em: http://www.who.int/healthacademy/ news/en/.

3. Topol E. The Cretive Destruition of Medicine. New York: Basic Books (AZ); 2011.

4. World Health Organization (WHO). Global Strategy on Digital Health [Internet]. 2020 [acessado $2020 \mathrm{mar}$ 24]. Disponível em: www.who.int/health-topics/digital-health\#tab=tab_1

5. Rocha TAH, Fachini LA, Thumé E, Silva NC, Barbosa ACQ, Carmo M, Rodrigues JM. Saúde Móvel: novas perspectivas para a oferta de serviços em saúde. Epidemiol Serv Saúde 2016; 25(1):159-170.

6. Organização das Nações Unidas (ONU). Transformando Nosso Mundo: A Agenda 2030 para o Desenvolvimento Sustentável [Internet]. 2015 [acessado 2020 jun 05]. Disponível em: http://svs.aids.gov.br/ dantps/acesso-a-informacao/acoes-e-programas/ods/ publicacoes/transformando-nosso-mundo-a-agenda-2030-para-o-desenvolvimento-sustentavel.pdf.

7. Centro Regional de Estudos para o Desenvolvimento da Sociedade da Informação (CETIC). TIC domicílios 2019: principais resultados [Internet]. 2020 [acessado 2020 jun 05]. Disponível em: https://cetic.br/media/ analises/tic_domicilios_2019_coletiva_imprensa.pdf

8. Santos GS, Pieszak GM, Gomes GC, Biazus CB, Silva SO. Contribuições da Primeira Infância Melhor para o crescimento e desenvolvimento infantil na percepção das famílias. Rev Fun Care Online 2019; 11(1):67-73.

9. Azevedo RFL, Morrow D, Graumlich J, WillemsenDunlap A, Hasegawa-Johnson M, Huang TS, Gu K, Bhat S, Sakakini T, Sadauskas V, Halpin DJ. Using conversational agents to explain medication instructions to older adults. AMIA Annu Symp Proc 2018; 2018:185-194.

10. Kimani E, Bickmore T, Trinh H, Ring L, Paasche-Orlow MK, Magnani JW. A smartphone-based virtual agent for atrial fibrillation education and counseling. IVA 2016; 10011:120-127.

11. Gabarron E, Larbi D, Denecke K, Årsand E. What Do We Know About the Use of Chatbots for Public Health? Stud Health Technol Inform 2020; 270:796-800.

12. Valter R, Santiago S, Andrade LOM, Barreto ICDHC. Data Mining and Risk Analysis Supporting Decision in Brazilian Public Health Systems. IEEE Adv Tecnol Humanit 2019; 1-6.

13. Gardini LM, Braga R, Bringel J, Oliveira C, Andrade $\mathrm{R}$, Martin H, Andrade LOM, Oliveira M. Clariisa, a context-aware framework based on geolocation for a health care governance system. Healthcom 2013 2013; 334-339.

14. Creswell JW. Projeto de pesquisa: métodos qualitativo, quantitativo e misto. Porto Alegre: Artmed; 2010.

15. Soares AVN, Gaidzinski RR. Carga de trabalho de enfermagem no Sistema de Alojamento Conjunto [Internet]. São Paulo: Biblioteca Digital de Teses e Dissertações da Universidade de São Paulo; 2009 [acessado 2019 ago 21]. Disponível em: http://www.teses.usp.br/ teses/disponiveis/7/7136/tde-14052009-115157/ 
16. Brasil. Ministério da Saúde (MS). Atenção à Saúde do Recém-Nascido. Brasília: MS; 2014.

17. Brasil. Ministério da Saúde (MS). Saúde da Criança: aleitamento materno e alimentação complementar. Brasília: MS; 2015.

18. Brasil. Ministério da Saúde (MS). Atenção Risco, Prénatal de Baixo. Brasília: MS; 2012.

19. Brasil. Ministério da Saúde (MS). Manual de Normase Procedimentos para Vacinação. Brasília: MS; 2014.

20. Reactive Native. Portal do Framework React Native [Internet]. [acessado 2019 jan 05]. Disponível em: https://www.reactnative.dev/.

21. Pallets Projects. Documentação do Framework Flask [Internet]. [acessado 2019 jan 03]. Disponível em: https://flask.palletsprojects.com/en/1.1.x/.

22. Loper E, Klein E, Bird S. Natural Language Processing with Python. Versão 3.0 [Internet]. [acessado 2019 out 10]. Disponível em: http://www.nltk.org/book/ ch00-pt.html.

23. Spizzirri RCP, Wagner A, Mosmann CP, Armani AB. Adolescência conectada: Mapeando o uso da internet em jovens internautas. Psicol Argumento 2017; 30(69):327-335.

24. Silva AG, Cruz O, Olinto G. Diferenças de gênero no uso das tecnologias da informação e da comunicação: um estudo na Biblioteca Parque de Manguinhos. In: XVI Encontro Nacional de Pesquisa em Ciência da Informação. João Pessoa; 2015.

25. Gomes HO. Inteligência artificial na saúde pública e privada é possível? Rev Cien Med Biol 2018; 17(3):285286.

26. Kumar VM, Keerthana A. Sanative Chatbot For Health Seekers. Int J Eng Comput Sci 2016; 05(16022):1602216025.

27. Ilić DT, Marković BM. Possibilities, limitations and economic aspects of artificial intelligence aplications in healthcare. Ecoforum 2016; 5(1):70-77.

28. Cameron G, Cameron D, Megaw G, Bond R, Mulvenna M, O'Neill S, Armour C, McTear M. Towards a chatbot for digital counselling. In: HCI 2017: Digital Make Believe - Proceedings of the 31st International BCS Human Computer Interaction Conference. BCS Learning and Development Ltd.; 2017.

29. Yadav D, Malik P, Dabas K, Singh P. FeedPal: Understanding opportunities for chatbots in breastfeeding education of women in India. Proc ACM Human-Computer Interact; 2019; 3(CSCW).

Artigo apresentado em 17/07/2020

Aprovado em 19/02/2021

Versão final apresentada em 21/02/2021

Editores-chefes: Romeu Gomes, Antônio Augusto Moura da Silva 